\title{
Non-perturbative Renormalization of Bilinear Operators on Fine Lattice
}

\author{
Hwancheol Jeong, Weonjong Lee, Jeonghwan Pak, Sungwoo Park \\ Lattice Gauge Theory Research Center, CTP, and FPRD, \\ Department of Physics and Astronomy, \\ Seoul National University, Seoul, 151-747, South Korea \\ E-mail: wlee@snu.ac.kr

\section{Jangho Kim*} \\ National Institute of Supercomputing and Networking, \\ Korea Institute of Science and Technology Information, Daejeon 34141, Korea \\ E-mail: jangho@kisti.re.kr
}

\section{SWME Collaboration}

We present results of the wave function renormalization factor $Z_{q}$ and mass renormalization factor $Z_{m}$ obtained using non-perturbative renormalization (NPR) method in the RI-MOM scheme with HYP improved staggered quarks. We use fine ensembles of MILC asqtad lattices $\left(N_{f}=2+1\right)$ with $28^{3} \times 96$ geometry, $a \approx 0.09 \mathrm{fm}$, and $a m_{\ell} / a m_{s}=0.0062 / 0.031$. We also study on scalability of $Z_{q}$ and $Z_{m}$ by comparing the results on the coarse and fine ensembles.

The 33rd International Symposium on Lattice Field Theory

14 - 18 July 2015

Kobe International Conference Center, Kobe, Japan

\footnotetext{
* Speaker.
} 


\section{Introduction}

In our previous work [1], we presented the results of the wave function renormalization factor $Z_{q}$, mass renormalization factor $Z_{m}$ and the complete set of renormalization factors for bilinear operators obtained on the $20^{3} \times 64$ MILC asqtad coarse lattice at $a \approx 0.12 \mathrm{fm}$ with $a m_{\ell} / a m_{s}=$ $0.01 / 0.05$. In this proceeding, we analyse the $Z_{q}$ and $Z_{m}$ on the $28^{3} \times 96$ MILC asqtad fine lattices $\left(a \approx 0.09 \mathrm{fm}, a m_{\ell} / a m_{s}=0.0062 / 0.031\right)$ and compare the results with those on the coarse lattices.

\section{Results}

We calculate the renormalization factors with Landau gauge fixing using HYP-smeared staggered quarks. To do the chiral extrapolation, we perform the measurements with 5 valence quark masses $(a m=0.0062,0.0124,0.0186,0.0248,0.031)$ on the MILC fine ensembles at $a \approx 0.09 \mathrm{fm}$. We also carry out the measurements for 20 external momenta given in Table 1 . The measurements are done over 30 gauge configurations.

\begin{tabular}{c|c|c||c|c|c||c|c|c}
\hline \hline$n(x, y, z, t)$ & $a|\widetilde{p}|$ & $\mathrm{GeV}$ & $n(x, y, z, t)$ & $a|\widetilde{p}|$ & $\mathrm{GeV}$ & $n(x, y, z, t)$ & $a|\widetilde{p}|$ & $\mathrm{GeV}$ \\
\hline$(1,1,1,3)$ & 0.4355 & 1.0197 & $(1,1,1,4)$ & 0.4686 & 1.0974 & $(1,2,1,4)$ & 0.6088 & 1.4257 \\
$(1,2,1,6)$ & 0.6755 & 1.5819 & $(2,1,2,6)$ & 0.7794 & 1.8250 & $(2,2,2,7)$ & 0.9023 & 2.1130 \\
$(2,2,2,8)$ & 0.9372 & 2.1947 & $(2,2,2,9)$ & 0.9753 & 2.2839 & $(2,3,2,7)$ & 1.0324 & 2.4177 \\
$(2,3,2,8)$ & 1.0631 & 2.4895 & $(2,3,2,9)$ & 1.0968 & 2.5684 & $(3,2,3,8)$ & 1.1756 & 2.7529 \\
$(3,3,3,7)$ & 1.2528 & 2.9337 & $(3,3,3,8)$ & 1.2782 & 2.9931 & $(3,3,3,10)$ & 1.3371 & 3.1312 \\
$(3,4,3,9)$ & 1.4349 & 3.3602 & $(4,3,4,10)$ & 1.5789 & 3.6973 & $(4,4,4,10)$ & 1.6868 & 3.9501 \\
$(4,4,4,12)$ & 1.7418 & 4.0788 & $(4,4,4,14)$ & 1.8046 & 4.2259 & & & \\
\hline \hline
\end{tabular}

Table 1: The list of momenta used for our analysis. The first column is the four vectors in the units of $\left(\frac{2 \pi}{L_{s}}, \frac{2 \pi}{L_{S}}, \frac{2 \pi}{L_{S}}, \frac{2 \pi}{L_{t}}\right)$, where $L_{s}\left(L_{t}\right)$ is the number of sites in the spatial (temporal) direction.

\subsection{Wave Function Renormalization Factor $Z_{q}$}

Let us consider the conserved vector current to obtain the wave function renormalization factor $Z_{q}$. We use the same method as in Ref. [1] to obtain the $Z_{q}$. First, we convert the raw data to the data defined at a common scale (CS) $\mu_{0}=3 \mathrm{GeV}$ using the four-loop RG evolution equation in Ref. [2, 3]. In Fig. 1, we present the raw data as the black circles and CS data as blue diamonds as a function of the square of reduced momentum $(a \widetilde{p})^{2}$ at a fixed quark mass $(a m=0.0062)$.

After converting the raw date to the CS data, we perform the fitting with respect to quark masses at a fixed external momentum to the following fitting function. We call this $\mathrm{m}$-fit.

$$
f_{\mathrm{m}-\mathrm{fit}}=b_{1}+b_{2} \cdot a m+b_{3} \cdot(a m)^{2}
$$

The fitting results are presented in Table 2 and the plot is given in Fig. 2(a).

We take $b_{1}$ as the chiral limit values which are function of external momentum $(a \widetilde{p})^{2}$. After $\mathrm{m}$-fit, we fit $b_{1}$ to the following fitting function. We call this $\mathrm{p}$-fit.

$$
f_{\mathrm{p}-\mathrm{fit}}=c_{1}+c_{2}(a \widetilde{p})^{2}+c_{3} \cdot\left((a \widetilde{p})^{2}\right)^{2}+c_{4} \cdot(a \widetilde{p})^{4}
$$




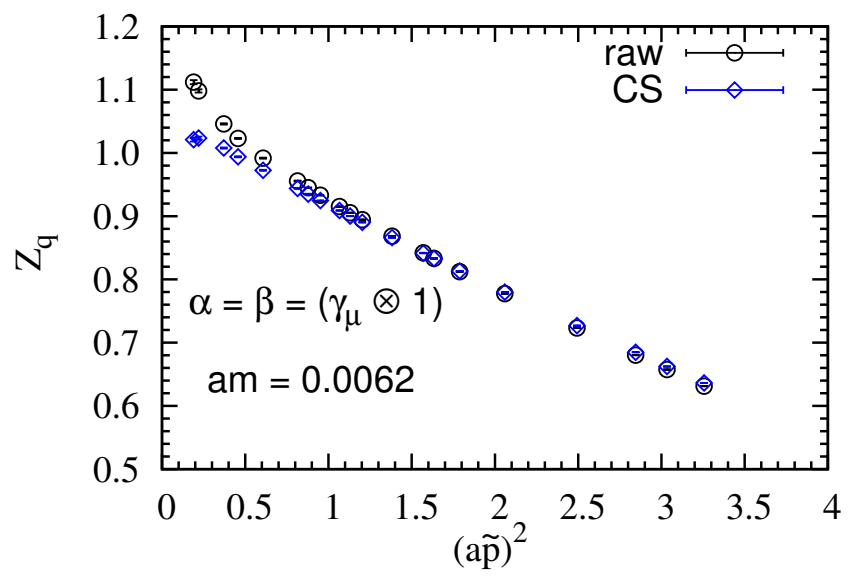

Figure 1: $Z_{q}$ obtained from conserved vector current $(V \times S)$ at a fixed quark mass $(a m=0.0062)$. The black circles represent raw data and blue diamonds are CS data at a CS $\mu_{0}=3 \mathrm{GeV}$.

\begin{tabular}{c|c|c||c}
\hline \hline$b_{1}$ & $b_{2}$ & $b_{3}$ & $\chi^{2} /$ dof \\
\hline $0.84141(15)$ & $0.0153(97)$ & $-0.31(17)$ & $0.004(10)$ \\
\hline \hline
\end{tabular}

Table 2: $\mathrm{m}$-fit results for $Z_{q}$ at $\mu_{0}=3 \mathrm{GeV}$ for a fixed external momentum $n=(3,3,3,7)$.

\begin{tabular}{c|c|c|c||c}
\hline \hline$c_{1}$ & $c_{2}$ & $c_{3}$ & $c_{4}$ & $\chi^{2} / \mathrm{dof}$ \\
\hline $1.0567(11)$ & $-0.1452(10)$ & $0.00294(14)$ & $0.0082(11)$ & $0.13(26)$ \\
\hline \hline
\end{tabular}

Table 3: P-fit results for $Z_{q}$ at $\mu_{0}=3 \mathrm{GeV}$.

The fitting results are presented in Table 3 and the plot is presented in Fig. 2(b). The $\mathscr{O}\left((a \widetilde{p})^{2}\right)$ and higher order terms correspond to lattice artifacts. Hence, we take $c_{1}$ as $Z_{q}$ value in RI-MOM scheme at $\mu_{0}=3 \mathrm{GeV}$. Using the four-loop RG running formula [2, 3], we convert the $Z_{q}$ from the RI-MOM scheme to the $\overline{\mathrm{MS}}$ scheme.

We estimate the systematic error in two different ways. One systematic error comes from truncation of four-loop RG running factor which is used to convert the $Z_{q}$ from the RI-MOM scheme to the $\overline{\mathrm{MS}}$ scheme. Hence, we take five-loop uncertainty $\left(\sim \mathscr{O}\left(\alpha_{s}^{4}\right)\right)$ and define $E_{t}$ as follows.

$$
E_{t}=Z_{q}^{\mathrm{RI}-\mathrm{MOM}} \cdot\left(\alpha_{s}\right)^{4}
$$

The other systematic error comes from the difference between the conserved vector and axial currents. Theoretically, $Z_{q}$ obtained from the conserved vector and axial currents must be identical to each other. However, they are not same in our study. Hence, we take the difference of them as the systematic error and define $E_{\Delta}$ as follows.

$$
E_{\Delta}=\left|Z_{q}(V \otimes S)-Z_{q}(A \otimes P)\right|
$$

The total error $\left(E_{\mathrm{tot}}\right)$ is obtained adding the statistical error $\left(E_{\mathrm{stat}}\right)$ and the systematic errors in 


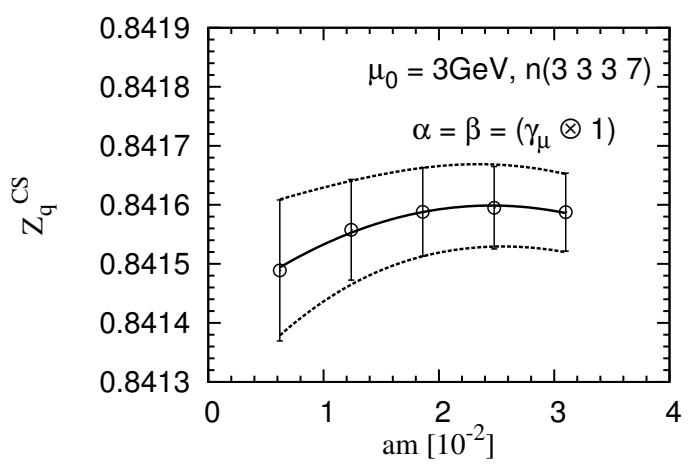

(a) m-fit for $Z_{q}$

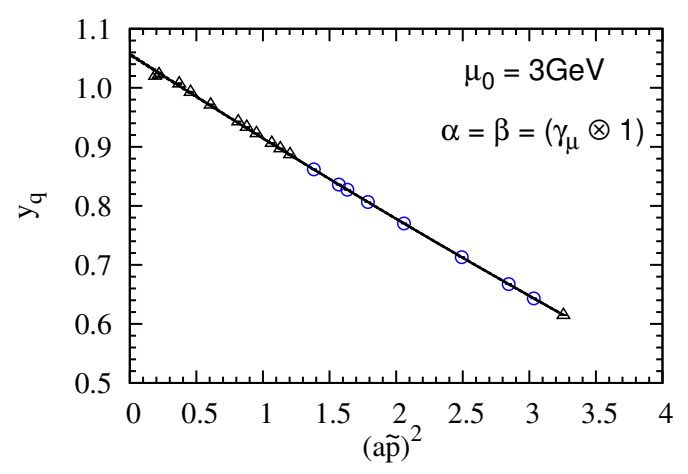

(b) p-fit for $Z_{q}$

Figure 2: (a) m-fit results for $Z_{q}$ at a reduced momentum $n=(3,3,3,7)$ and (b) p-fit results for $y_{q}$. Here, we use the conserved vector current at $\mu_{0}=3 \mathrm{GeV} . y_{q} \equiv Z_{q}\left(\mu_{0}, a m=0\right)-\left\langle c_{4}\right\rangle(a \tilde{p})^{4}$. The blue circles are used for fitting.

quadrature. We present the final result of $Z_{q}$ in $\overline{\mathrm{MS}}$ scheme at $\mu_{0}=3 \mathrm{GeV}$ and its statistical and systematic errors in Table 4.

\begin{tabular}{c||c|c|c|c}
\hline \hline$Z_{q}^{\mathrm{MS}}\left(\mu_{0}\right)$ & $E_{\text {stat }}$ & $E_{t}$ & $E_{\Delta}$ & $E_{\text {tot }}$ \\
\hline 1.0494 & 0.0011 & 0.0038 & 0.0099 & 0.0107 \\
\hline \hline
\end{tabular}

Table 4: $Z_{q}$ in the $\overline{\mathrm{MS}}$ scheme at $\mu_{0}=3 \mathrm{GeV}$ with statistical and systematic errors.

\subsection{Quark Mass Renormalization Factor $Z_{m}$}

Quark mass renormalization factor $Z_{m}$ is obtained from the bilinear operator $[S \otimes S]$. Here, we use the same analysis method as in Ref. [1]. Note that we analyse $Z_{q} \cdot Z_{m}$ instead of $Z_{m}$ directly. After we obtain the $Z_{q} \cdot Z_{m}$ in RI-MOM scheme at $\mu_{0}=3 \mathrm{GeV}$ through m-fit and p-fit, we divide by $Z_{q}$ obtained from the conserved vector current. First, we convert raw data to the CS data using the four-loop RG running formula for $Z_{q} \cdot Z_{m}$. We present the raw and CS data for $Z_{q} \cdot Z_{m}$ in Fig. 3 .

Using the CS data for $Z_{q} \cdot Z_{m}$, we carry out $\mathrm{m}$-fit and $\mathrm{p}$-fit. The fitting functions for $\mathrm{m}$-fit and p-fit are

$$
\begin{aligned}
& g_{\mathrm{m}-\mathrm{fit}}=d_{1}+d_{2} \cdot a m+d_{3} \cdot \frac{1}{(a m)^{2}} \\
& g_{\mathrm{p}-\mathrm{fit}}=h_{1}+h_{2}(a \widetilde{p})^{2}+h_{3} \cdot\left((a \widetilde{p})^{2}\right)^{2}+h_{4} \cdot(a \widetilde{p})^{4} .
\end{aligned}
$$

We present fitting results of $\mathrm{m}$-fit in Table 5 and in Fig. 4 (a). We show fitting results of p-fit in Table 6 and in Fig. 4 (b).

We determine $Z_{m}$ by dividing $Z_{q} \cdot Z_{m}$ by $Z_{q}$ obtained using the conserved vector current. Then, we convert $Z_{m}$ in the RI-MOM scheme into that in the $\overline{\mathrm{MS}}$ scheme using the four-loop RG evolution formula.

$$
Z_{m}^{\overline{\mathrm{MS}}}\left(\mu_{0}\right)=U\left(\infty \rightarrow \mu_{0}, \overline{\mathrm{MS}}\right) U\left(\mu_{0} \rightarrow \infty, \mathrm{RI}-\mathrm{MOM}\right) Z_{m}^{\mathrm{RI}-\mathrm{MOM}}\left(\mu_{0}\right),
$$




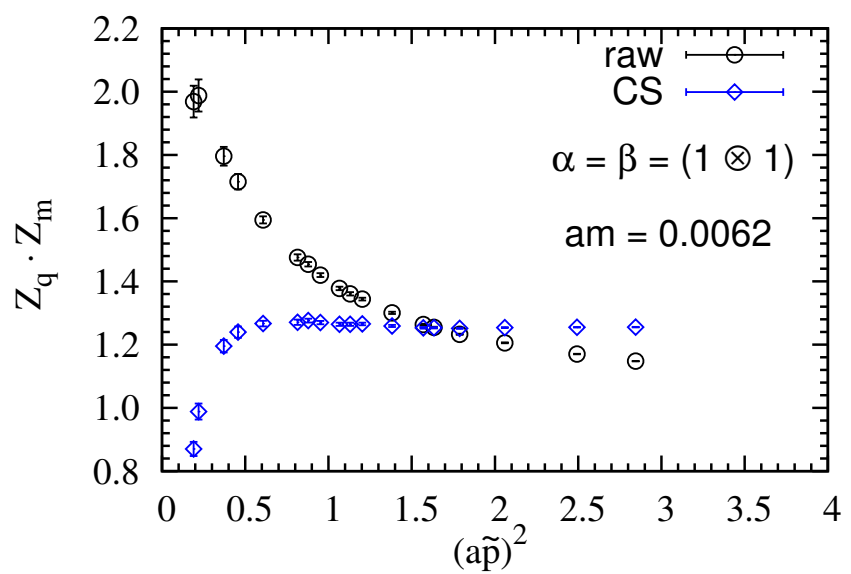

Figure 3: $Z_{q} \cdot Z_{m}$ obtained from $[S \times S]$ bilinear operator at am $=0.0062$. Here, $\mu_{0}=3 \mathrm{GeV}$.

\begin{tabular}{c|c|c||c}
\hline \hline$d_{1}$ & $d_{2}$ & $d_{3}$ & $\chi^{2} /$ dof \\
\hline $1.25664(60)$ & $-0.354(15)$ & $-0.000000019(51)$ & $0.017(25)$ \\
\hline \hline
\end{tabular}

Table 5: Fitting results of $Z_{q} \cdot Z_{m}$ for $\mathrm{m}$-fit. The reduced momentum is fixed to $n=(3,4,3,9)$.

\begin{tabular}{c|c|c|c||c}
\hline \hline$h_{1}$ & $h_{2}$ & $h_{3}$ & $h_{4}$ & $\chi^{2} /$ dof \\
\hline $1.3069(39)$ & $-0.0459(35)$ & $0.00191(62)$ & $0.0308(45)$ & $0.37(32)$ \\
\hline \hline
\end{tabular}

Table 6: Fitting results of $Z_{q} \cdot Z_{m}$ for p-fit.

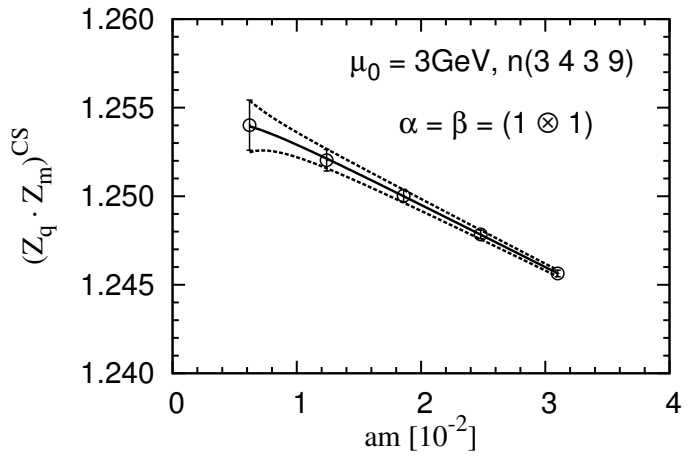

(a) m-fit

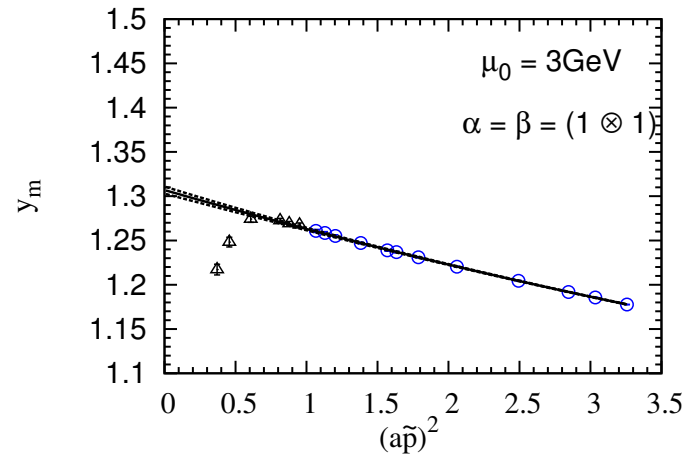

(b) p-fit

Figure 4: Fitting results of $Z_{q} \cdot Z_{m}$ for (a) m-fit and (b) p-fit. For the m-fit, the reduced momentum is fixed to $n=(3,4,3,9)$. For the p-fit, $y_{m} \equiv\left(Z_{q} \cdot Z_{m}\right)\left(\mu_{0}, a m=0\right)-\left\langle h_{4}\right\rangle(a \widetilde{p})^{4}$. The blue circle data are used for fitting.

where $U\left(\mu_{1} \rightarrow \mu_{2}, R\right)$ is the RG evolution matrix from the scale $\mu_{1}$ to $\mu_{2}$ in the $R$ scheme. The results are summarized in Table 7 . Here, the systematic errors are estimated in the same way as in $Z_{q}$. 


\begin{tabular}{c||c|c|c|c}
\hline \hline$Z_{m}^{\overline{\mathrm{MS}}}\left(\mu_{0}\right)$ & $E_{\text {stat }}$ & $E_{t}$ & $E_{\Delta}$ & $E_{\text {tot }}$ \\
\hline 1.0117 & 0.0032 & 0.0044 & 0.0005 & 0.0055 \\
\hline \hline
\end{tabular}

Table 7: $Z_{m}$ in $\overline{\mathrm{MS}}$ scheme at $\mu_{0}=3 \mathrm{GeV}$.

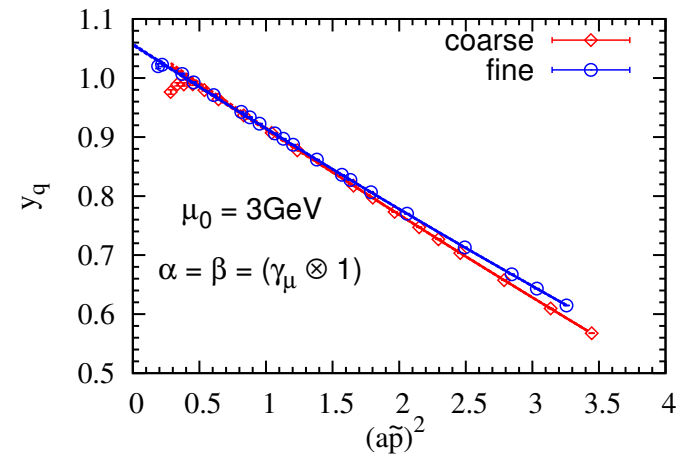

(a) p-fit

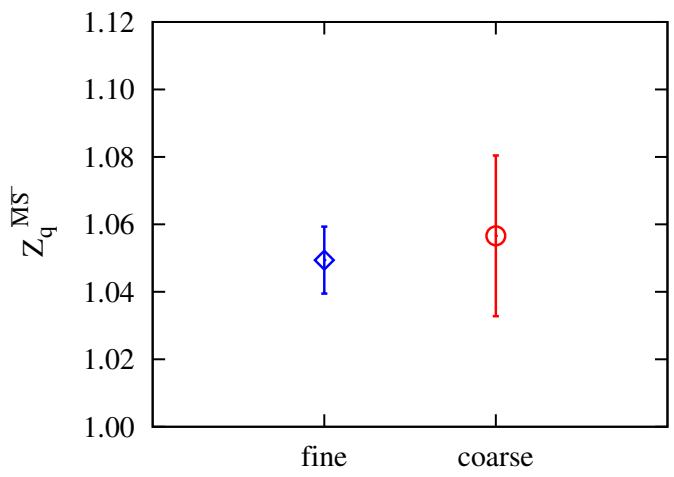

(b) final results

Figure 5: Comparison of $Z_{q}\left(\mu_{0}\right)$ on the coarse and fine lattices. The red (blue) data represent results on the coarse (fine) lattice.

\subsection{Comparison of $Z_{q}$ and $Z_{m}$ between coarse and fine lattices}

Now, we present the comparison of the $Z_{q}$ and $Z_{m}$ results at $\mu_{0}=3 \mathrm{GeV}$ between coarse and fine lattices in Fig. 5 and Fig. 6.

The results of $Z_{q}$ and $Z_{m}$ at $\mu_{0}=3 \mathrm{GeV}$ on coarse and fine lattices are presented in Table 8 . We find that the total errors of $Z_{q}^{\overline{\mathrm{MS}}}$ and $Z_{m}^{\overline{\mathrm{MS}}}$ on fine lattice are reduced dramatically compared with those of coarse lattice.

\begin{tabular}{c||c|c}
\hline & coarse & fine \\
\hline \hline$Z_{q}^{\overline{\mathrm{MS}}}(3 \mathrm{GeV})$ & $1.0566(59)(231)$ & $1.0494(11)(99)$ \\
\hline$Z_{m}^{\mathrm{MS}}(3 \mathrm{GeV})$ & $0.865(21)(25)$ & $1.0117(32)(44)$ \\
\hline
\end{tabular}

Table 8: The comparison of $Z_{q}$ and $Z_{m}$ at $\mu_{0}=3 \mathrm{GeV}$ between coarse and fine lattices. The first error is statistical and the second is systematic.

\section{Conclusion}

Here, we present the results of the wave function renormalization factor $Z_{q}$ and mass renormalization factor $Z_{m}$ for the staggered bilinear operators defined in the $\overline{\mathrm{MS}}$ scheme at $\mu_{0}=3 \mathrm{GeV}$. We use the NPR method in the RI-MOM scheme as an intermediate scheme. We use one of the MILC asqtad fine $(a \approx 0.09 \mathrm{fm})$ ensembles to calculate the matching factors. By comparing results with those on the coarse ensembles, we find that the statistical and systematic errors of $Z_{q}$ and $Z_{m}$ are reduced dramatically on the fine lattice. We plan to extend the calculation to the superfine $(a \approx 0.06 \mathrm{fm})$ and ultrafine $(a \approx 0.045 \mathrm{fm})$ ensembles in the future. As a consequence, we will 


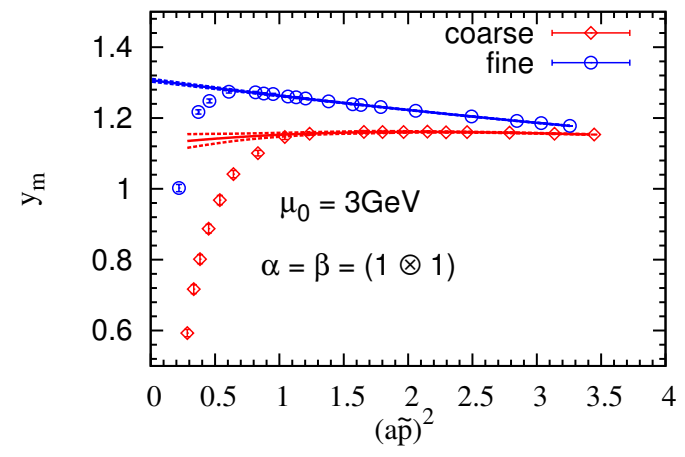

(a) p-fit

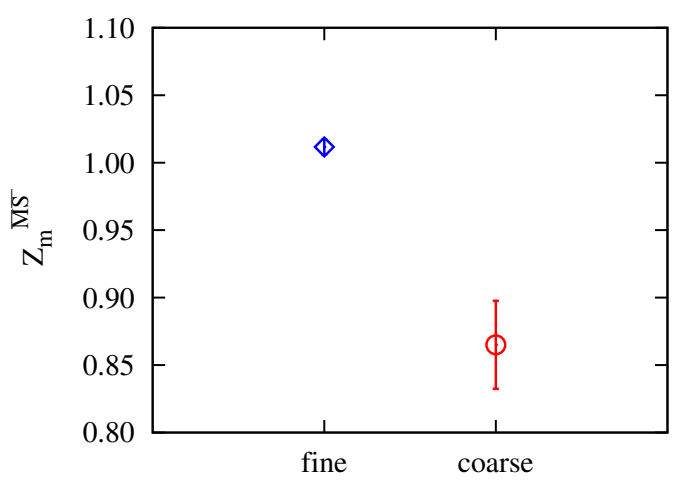

(b) final results

Figure 6: Comparison of $Z_{m}\left(\mu_{0}\right)$ on the coarse and fine lattices. The red (blue) data represent results on the coarse (fine) lattice.

study on the scalability of $Z_{q}$ and $Z_{m}$. We also plan to calculate the renormalization factors on the fine ensembles with different sea quark masses, which will help us to understand their dependence on sea quark masses.

\section{Acknowledgments}

J. Kim is supported by Young Scientists Fellowship through National Research Council of Science \& Technology (NST) of KOREA. The research of W. Lee is supported by the Creative Research Initiatives Program (No. 2015001776) of the NRF grant funded by the Korean government (MEST). W. Lee would like to acknowledge the support from the KISTI supercomputing center through the strategic support program for the supercomputing application research (No. KSC-2014G3-002) with much gratitude. Computations were carried out on the DAVID GPU clusters at Seoul National University.

\section{References}

[1] J. Kim, J. Kim, W. Lee, and B. Yoon PoS LATTICE2013 (2013) 308, [1310 . 4269].

[2] K. Chetyrkin and A. Retey, Renormalization and running of quark mass and field in the regularization invariant and MS-bar schemes at three loops and four loops, Nucl.Phys. B583 (2000) 3-34, [hep-ph/9910332].

[3] Y. Aoki, P. Boyle, N. Christ, C. Dawson, M. Donnellan, et al. Phys.Rev. D78 (2008) 054510, [0712.1061]. 